\title{
The Relationship between Social Support and Depression in University students: The Meaning in Life as Mediation
}

\author{
Heyan Qing* and Shang Li \\ Department of Education, Yunnan Normal University, 650500, Kunming, China
}

\begin{abstract}
This study aims to explore the relationship between social support, meaning in life and depression, and further explore the mediating role of meaning in life between social support and depression. This survey of 287 university students were investigated with the short version of Center for Epidemiologic Studies Depression Scale (CES-D-13), the Social Support Rating Scale (SSRS), and the Chinese Meaning in Life Questionnaire (MLQ). The results showed that: (1)The junior is the most serious period of depressive symptoms(11.33 \pm 7.019$)$, and also an important turning point; (2) Non-only-child (10.23 \pm 6.713 ) were significantly higher than the only-child $(9.02 \pm 6.230)$ about depressive symptoms $(p=0.0368)$; (3)The depression were significantly negatively correlated with social support $(\mathrm{r}=-0.35, p<0.01)$ and the meaning in life $(\mathrm{r}=-$ $0.39, \mathrm{p}<0.01$ ), but there was a significant negative correlation between the meaning in life and social support $(\mathrm{r}=0.28, p<0.01)$; (4) The meaning in life plays a partial mediating role between social supports and depressive symptoms in university students. This research provides theoretical evidence for the prevention and intervention of university students' depression from two aspects of social support and meaning in life.
\end{abstract}

\section{Introduction}

Depression is a mood disorder which is manifested as significant and enduring depression, drops of interest, anhedonia and other clinical manifestations, characterized by high morbidity, high recurrence rate, high suicide rate and high disability rate [1]. The pathogenesis of depression is complex, and its formation is closely related to long-term chronic events and experiences that threaten and challenge the physically or psychologically [2]. It brings certain pain and damage to the individual's life, study and work. Depression is a common mental health problem among university students. Under the background of education system reform and grim employment situation, university students are facing the increasingly pressure in study and employment, thus the depressive symptoms are prone to show up [3].For example, Wang et al. conducted a meta-analysis on the survey results of the prevalence of depression among Chinese university students from

* Corresponding author: 2928982287@qq.com 
2009 to 2019, and found that the prevalence of depression was increasing [4]. On account of the psychosomatic harm caused by depressive symptoms and the increasing number among university students, universities should attach great attention to the influencing factors and mechanism of depression in university students, which is of great significance for the healthy growth of university students.

According to the Interpersonal Model, individuals with low social support level are prone to get depression when they are facing with stressful life events [5]. Also, the internal mechanisms of social support in relation to depression in adolescents include main effect and stress buffering effect. The former emphasizes that good social support is beneficial to individuals no matter whether they are in a state of stress or not; The latter emphasizes that social support can alleviate the negative impact of individual stressful events and reduce the incidence of depression [6]. Social support refers to the spiritual and material support obtained from the social relations of family members, relatives and friends, colleagues, groups, organizations and communities [7]. Therefore, social support is one of the main factors affecting individual depression. There is a stable moderate negative correlation between social support and depression among Chinese university students [8,9], but they are not directly related with each other. So further understanding about how social support affects the mediating variables of depression is helpful for universities to formulate measures for prevention and intervention of depression.

Steger et al. divided the meaning of life into the experience of the meaning in life and the seeking of the meaning in life [10]. Studies have shown that the higher the level of social support is, the more conducive to improving university students' sense of the meaning in life [11]. However, individuals who are lacking in the sense of the meaning in life will lead to depression. Individual with higher the meaning in life had lower depressive symptoms. However, individuals with low the meaning in life have higher depression scores [12-15]. It is speculated that social support may indirectly affect depression by affecting the sense of meaning in life.

Existing studies have found that strengthening social support can reduce depression, and the increase of sense of meaning in life can also reduce depression. But three is no research on the mechanism among the three. To sum up, this study will investigate the influence mechanism of the meaning in life and social support on depression through questionnaire survey, and test hypothesis H1: the meaning in life plays a mediating role between social support and university students' depression.

\section{Methods}

\subsection{Participants}

A total of 287 valid questionnaires were returned from two university in Yunan provinces. Among the respondents, 134 males (46.7\%) and 153 females (53.3\%); freshmen 71(24.7\%) and sophomores 82(28.6), juniors 89(31.0\%), and senior 45(15.7\%); only-child 61(21.3\%) and non-only-child 226(78.7\%).

\subsection{Measures}

\subsubsection{Social Support Rating Scale (independent variable)}

Social Support Rating Scale (SSRS) compiled by Xiao and Yang [16], has 10 items, which is divided into 3 dimensions: Subjective social support $(1,3,4,5)$, objective support 
dimension $(2,6,7)$ and the dimension of the utilization of support $(8,9,10)$. Considering the actual situation of the subjects, some items in the scale were revised. For example, in question 4, "colleague" was changed to "classmate". In this scale, items of 1-4 and 8-10 are single choices, and choosing A, B, C and D are scored 1-4 points respectively. Item 5 A, B, $\mathrm{C}$ and $\mathrm{D}$ will be given A total score, with 1-4 points for each item from none to full support. For question 7 and 8, a response to "No source" will be awarded 0 points, and one point for each source will be awarded 1 point. The higher scores represented the better social support. In this study, the Cronbach's $\alpha$ was 0.67 .

\subsubsection{Meaning in Life (Mediation)}

Meaning in Life Questionnaire MLQ compiled by American scholar Steger, etc. [10], Wang and Dai [17] the questionnaire is revised, a total of ten projects, can be divided into two dimensions: Experience of meaning (questions 1, 4, 5, 6, 9) and pursuit of meaning (questions 2, 3, 7, 8, 10). The scale has 10 items, which is a 7-point scale, with the options ranging from $1=$ "Completely agree" to $7=$ "Completely disagree". Three items in the positive emotion dimension were scored in reverse. The higher the scores represented the stronger meaning in life. In this study, the Cronbach's $\alpha$ was 0.87 .

\subsubsection{Depression}

This scale was revised by Li and his colleagues and obtained the short version of Center for Epidemiologic Studies Depression Scale (CES-D-13) based on several simplified versions of CES-D commonly used abroad [18]. The scale has 13 items, which is a 4-point scale, divided into 3 dimensions: Somatic symptoms, depressive emotions, and positive emotions. $0=$ "occasionally or none", $1=$ "sometimes", 2="often or half the time", 3="Most of the time or duration". Three items in the positive emotion dimension were scored in reverse. The higher the scores represented the more depressive symptoms. In this study, the Cronbach's $\alpha$ was 0.86 .

\subsection{Data Analysis}

SPSS22.0 software was used for describe statistics, independent sample t-test, analysis of ANOVA, correlation analysis and mediating effect analysis.

\section{Results}

\subsection{Common method deviation test}

Exploratory factor analysis was conducted on all items in the three questionnaires, and the results showed that there were 13 factors with eigenvalues greater than 1 , among which the first factor could explain $16.91 \%$ of the total variation and was less than $40 \%$ of the critical value, indicating that there was no serious common method deviation in this study [19].

\subsection{Depression of University Students}

Taking "gender”, "grade” and "only child or not” as independent variable respectively and depression as dependent variable, the independent sample $t$-test and single factor analysis of ANOVA were conducted. The results showed that the junior is an important turning point. 
depressive symptoms increased before junior, and then decreased. After multiple comparisons found that there was a significant difference between junior and freshman $(p=0.016)$. Only children and the non-only-child significant differences on depressive symptoms ( $p=0.0368)$, non-only-child $(10.23 \pm 6.713)$ is higher than the only-child $(9.02 \pm 6.230)$ depressive symptoms. There was no significant difference in other ways.

Table1. Depression of different types of university Students

\begin{tabular}{|c|c|c|c|c|c|}
\hline \multicolumn{2}{|c|}{ Type } & $\mathbf{N}$ & $\mathbf{M} \pm \mathbf{S D}$ & $\mathbf{F}$ & $\mathbf{p}$ \\
\hline \multirow{2}{*}{ gender } & male & 134 & $9.55 \pm 6.195$ & \multirow{2}{*}{2.949} & \multirow{2}{*}{.087} \\
\hline & female & 153 & $10.35 \pm 6.973$ & & \\
\hline \multirow{4}{*}{ grade } & freshman & 71 & $8.79 \pm 5.299$ & \multirow{4}{*}{2.151} & \multirow{4}{*}{.094} \\
\hline & sophomore & 82 & $9.54 \pm 7.027$ & & \\
\hline & junior & 89 & $11.33 \pm 7.019$ & & \\
\hline & senior & 45 & $9.98 \pm 6.674$ & & \\
\hline \multirow{2}{*}{ Only child or not } & Only child & 61 & $9.02 \pm 6.230$ & \multirow{2}{*}{.812} & \multirow{2}{*}{$.0368 *$} \\
\hline & non-only-child & 226 & $10.23 \pm 6.713$ & & \\
\hline \multicolumn{6}{|c|}{ Note. $N=287 \quad{ }^{*} p<0.05 .{ }^{* *} p<0.01 .{ }^{* * *} p<0.001$} \\
\hline
\end{tabular}

\subsection{Describe statistical and correlation analyses of depression, social support and description of the meaning in life in university students}

The descriptive statistics and correlation analysis of the three study variables, depression, social support and the meaning in life, are shown in Table 2 . The results showed that there was a significant positive correlation between social support and the meaning in life $(r=0.28$, $\mathrm{p}<0.01)$, a significant negative correlation between social support and depression $(\mathrm{r}=-0.35$, $\mathrm{p}<0.01$ ), and a significant negative correlation between the meaning in life and depression $(\mathrm{r}=-0.39, \mathrm{p}<0.01)$.

Table2. Analysis of the relationships between depression, social support and life in meaning

\begin{tabular}{|l|c|c|c|c|c|}
\hline & Average & SD & $\mathbf{1}$ & $\mathbf{2}$ & $\mathbf{3}$ \\
\hline 1.social support & 34.17 & 5.18 & 1 & & \\
\hline 2.life in meaning & 50.59 & 10.38 & $.28^{* *}$ & 1 & \\
\hline 3.depression & 9.98 & 6.62 & $-.35^{* *}$ & $-.39^{* *}$ & 1 \\
\hline
\end{tabular}

\subsection{Mediating effect test of the meaning in life}

According to the mediating effect procedure tested by Wen and Ye [20], regression analysis was used to explore the mediating effect of the meaning in life on social support and depressive symptoms. In the first step, depression was used as the dependent variable and social support as the independent variable for regression analysis to obtain the path 
coefficient c. The second step takes the meaning of life as the dependent variable and takes social support as the independent variable to conduct regression analysis to get the path coefficient a; The third step takes depression as the dependent variable and social support and the meaning in life as the independent variables to conduct regression analysis to obtain path coefficients b and c'. The results showed that the regression coefficient of the first step regression equation $(t=-6.351, p<0.001)$, the regression coefficient of the second step regression equation $(\mathrm{t}=4.990, \mathrm{p}<0.001)$ and the regression coefficient of the intermediary variable of the third step regression equation $(\mathrm{t}=5.731, \mathrm{p}<0.001)$, the $\mathrm{t}$-test results showed that there was statistical significance. Here mediating effect is existed. The regression coefficient of social support to depression $(t=-6.351, \mathrm{p}<0.001)$ was still significant and ab and $c^{\prime}$ had the same sign, suggesting that the meaning in life played a partial mediating role between social support and depression. Through calculation, the mediating effect of the meaning in life accounted for $25.34 \%$ of the total effect, that is, social support can affect depression through influencing the mediating variable of the meaning in life (see Table 3 and Fig.1).

Table 3. The Mediating Role of the meaning in life in Social Support and Depression

\begin{tabular}{|c|c|c|c|c|c|c|}
\hline $\begin{array}{c}\text { Dependent } \\
\text { Variable }\end{array}$ & $\begin{array}{c}\text { Independent } \\
\text { Variable }\end{array}$ & $\mathbf{R}$ & $\mathbf{R}^{2}$ & $\mathbf{F}$ & $\boldsymbol{\beta}$ & $\mathbf{t}$ \\
\hline Dependent & Social support & .352 & .121 & 40.334 & -.352 & $-6.351^{* * *}$ \\
\hline Life in meaning & Social support & .283 & .077 & 24.905 & .283 & $4.990^{* * *}$ \\
\hline \multirow{2}{*}{ Depression } & Social support & .463 & .209 & 38.839 & -.263 & $-4.797^{* * *}$ \\
\cline { 2 - 7 } & Life in meaning & & & & -.314 & $-5.731^{* * *}$ \\
\hline
\end{tabular}

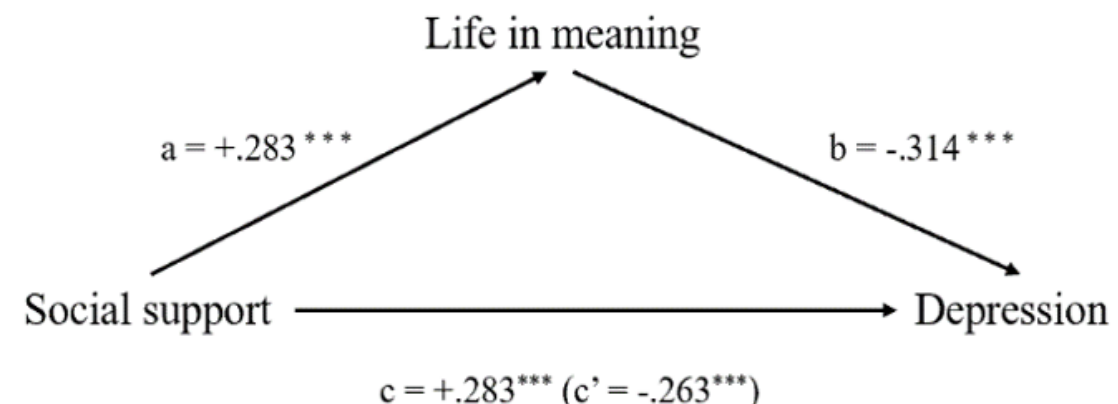

Fig.1 Mediating effect of life in meaning between social support and depression.

\section{Discussions}

\subsection{The relationship among social support, life in meaning and depression of the university Students}

The results of this study indicate that social support has a significant negative predictive effect on depression. This result is consistent with previous research [8,21]. In other words, individuals with high levels of social support are less likely to get depression, while individuals with low levels of social support, depression is more likely to arise. People with high levels of social support are more likely to experience emotional and material help from others, and get more positive emotions, so that the incidence of depressive symptoms will 
be reduced. It is proved that high level of social support can effectively prevent the occurrence of depressive symptoms to a certain extent.

In terms of the relationship between social support and the meaning in life, our results show that there is a significant positive correlation between social support and the meaning in life. This result is agreed with the research results of Chen et al. [11] on university students. The lower the level of social support represents the vaguer in the meaning in life is; The higher the level of social support represents the clearer the meaning in life is.

In terms of the relationship between depression and the meaning in life, the results of this study indicate that there is a significant negative correlation between the two. This result is consistent with previous research results [15]. For the individuals with less clear sense of the meaning in life, the more likely to produce depressive symptoms; The individuals with a clearer sense of the meaning in life showed fewer depressive symptoms.

\subsection{The relationship between social support and depression was partially mediated by meaning in life}

In the relationship between social support and depression, the meaning in life played a partial mediating role, and the mediating effect accounted for $25.34 \%$ in the total effect, which means social support affected depression through the mediating variable of the meaning in life. Individuals with a strong sense of the meaning in life have better mental health [15].And in clinical Frankl "meaning treatment" (logotherapy) and "meaning of the center for consultation and therapy" (a fancy-centered counseling and therapy), the improvement of the meaning of life is of positive advantage to the treatment for individuals who are in depression [22].As a mediating variable, the sense of the meaning in life can reduce the negative influence of social support on university students' emotions, so as to promote university students' mental health. On the one hand, the level of social support is related to whether the spiritual and material needs of university students are met, which directly impacts on the depression of university students. On the other hand, the changes of university students' sense of the meaning in life will also change the effect of social support on depression. Low sense of meaning in life and low level of social support will lead to poor mental health of university students, which will make university students' depression more serious. Therefore, when the level of social support cannot be changed, the negative impact of low level of social support on university students' psychology can be reduced by improving their sense of the meaning in life.

\section{Conclusions}

This study concluded that social support can directly and negatively predict university students' depression, and it can also indirectly predict university students' depression through the meaning in life.

According to the results, prevention and intervention of College Students' depressive symptoms are suggested as follows: Firstly, more attention should be paid to the defense and intervention of depression for the junior and non-only-child. Secondly, improve university students social support. Through friends, family, school and society to jointly create a good supporting environment, improve the ability of college students to adapt to adversity and resist pressure, to reduce the occurrence of depression has a certain role in avoiding. Thirdly, helping university students understand the meaning of life. Enhance the sense of life meaning through group counseling, death course education, meaning photography and other methods [23], so as to help college students find their own life meaning and then reduce the occurrence of depression. 


\section{References}

1. M. Miret, J.L. Ayuso-Mateos, J. Sanchez-Moreno \& E. Vieta, Neuroscience and Biobehavioral Reviews, Depressive disorders and suicide: Epidemiology, risk factors, and burden, 37(10), 2372-2374, 2013

2. Y. Liu, J. Liu, Y.S. Wu, Y.L. Chen, S.S. Ren, Y.W. Shang, Q.W. He \& Y.Z. Sang, Chinese Journal of Experimental Traditional Medical Formulae, Research Progress on The Mechanism of Neuronal Autophagy in Depression and The Regulatory Effect of Traditional Chinese Medicine, 1-11[2021-07-19]. https://doi.org/10.13422/j.cnki.syfjx.20211502, 2021

3. S.J. Lui, Y.Q. Hu, H.L. Sun, Chinese Journal of Clinical Psychology, Mediating Effect of Neuroticism and Coping Styles between Stress and Depression, 26(06), 12301233+1229, 2018

4. M.Y. Wang, J. Liu, X. Wu, L. Li, X.D. Hao, Q. Shen, M.T. Huang \& R. H. Sun, Journal of Hainan Medical University, The prevalence of depression among students in Chinese universities over the past decade: A mate-analysis, 26(09), 686-693+699, 2020

5. S. Cohen, T.A. Wills, Psychological Bulletin, Stress, social support, and the buffering hypothesis, 98(02), 310-357, 1985

6. Y.W. Qiu, Y.X. Lou, Y. Lei, Psychological Development and Education, Depression in Adolescent: A Perspective Based on Social Support, 37(02):288-297, 2021

7. X. Liu, X. Huang, Psychological Research, Social support and its mechanism for mental health, 3(01), 3-8+15,2010

8. L.X Cui, J. Liu, X.J. Luo, Journal of Psychological Science, The Mediating R ole of Positive Affect and Proactive Coping between Social Support and Depression, 38(04), 980-984, 2014

9. C.X. Yang, D.J. Zhang, Y.H. Liang \& T.Q. Hu, Chinese Mental Health Journal, Relationship between social support and depression among college students in China: A meta-analysis, 30(12), 939-945, 2016

10. M.F. Steger, P. Frazier, S. Oishi \& M. Kaler, Journal of Counseling Psychology, The meaning in life questionnaire: Assessing the presence of and search for meaning in life, 53(1), 80-93, 2006

11. Q.T. Chen, X.Q. Li, China Journal of Health Psychology, Relationships between the Meaning in Life, Social Support, Locus of Control and Subjective Well-being among College Students, 23(01), 96-99, 2015

12. N. Mascaro, H.R. David, Journal of Humanistic Psychology, The Role of Existential Meaning as a Buffer Against Stress, 73(4), 985-1014, 2005

13. N. Mascaro, H.R. David, Journal of Humanistic Psychology, The Role of Existential Meaning as a Buffer Against Stress, 46(2), 168-190, 2006

14. M.F. Steger, J.R. Mann, P. Michels \& T.C. Cooper, Journal of Psychosomatic Research, Meaning in life, anxiety, depression, and general health among smoking cessation patients, 67(4), 353-358, 2009

15. G. Kleftaras, E. Psarra, Psychology, Meaning in Life, Psychological Well-Being and Depressive Symptomatology: A Comparative Study, 3(4), 337-345, 2012

16. S.Y. Xiao, D.S. Yang, Chinese Mental Health Journal, The influence of social support on physical and mental health, (04), 183-187, 1987 
17. M.C. Wang, X.Y. Dai, Chinese Journal of Clinical Psychology, Chinese Meaning in Life Questionnaire Revised in College Students and Its Reliability and Validity Test, (05), 459-461, 2008

18. B.S. Zhang, J. Li, Chinese Mental Health Journal, Reliability and validity of a short version of Center for Epidemiologic Studies Depression Scale in national adult population, 25(7), 506-506, 2011

19. H. Zhao, L. Long, Advances in Psychological Science, Statistical Remedies for Common Method Biases, (06), 942-950, 2004

20. Z.L. Wen, B.J. Ye, Advances in Psychological Science, Analyses of Mediating Effects: The Development of Methods and Models, (05), 731-745, 2014

21. L. Pang, W. Hong, N. Zhang \& J. Yin, Chinese Journal of Clinical Psychology, Mediating Effect of Self-perception on the Relationship Between Social Support and Depression, 19(03), 352-354+326, 2011

22. P.T.P. Wong, Taylor and Francis, The Human Quest for Meaning: Theories, Research, and Applications, 06-19, 2013

23. X.J. Huang, X. Gao, X.Y. Chen, R.J. Chen \& J. Wu, China Journal of Health Psychology, Research progress in influence factors and interventions of meaning in life of college students, 28(12), 1900-1905, 2020 